\title{
Malleable Games - A Literature Review on Communities of Game Modders
}

\author{
Sarah-Kristin Thiel \\ Department of Computer Science, Aarhus Universitet \\ Aarhus, Denmark \\ thiel@cs.au.dk
}

\author{
Peter Lyle \\ Department of Computer Science, Aarhus Universitet \\ Aarhus, Denmark \\ peter.lyle@cs.au.dk
}

\begin{abstract}
The creations of people who modify games (game "modders") have had an significant impact on economics of game development. Being part of a creative participatory culture, their mods serve as a source of inspiration that has even steered the direction of game development in the past. This paper presents the findings of a comprehensive literature review on game modding. We structure our findings according to the three perspectives from which game modding has been analyzed: an outside perspective, a meta-level, and an inside perspective. We identify aspects that have received little academic attention so far, but warrant future research. Our analysis of the body of literature shows that only few publications focus more closely on community aspects such as differences among individual game modding communities, dynamics and collaboration within modder communities. We argue that gaining a deeper understanding of these aspects is essential in order to be able to capitalize from the huge creative potential that lies within game modding communities.
\end{abstract}

\section{CCS CONCEPTS}

- Human-centered computing $\rightarrow \mathrm{HCI}$ design and evaluation methods; Empirical studies in HCI; Ethnographic studies; • Software and its engineering $\rightarrow$ Open source model; $\bullet$ Applied computing $\rightarrow$ Collaborative learning;

\section{KEYWORDS}

communities, modding, game mods, games, literature review, modders, motivation, creativity

\section{ACM Reference Format:}

Sarah-Kristin Thiel and Peter Lyle. 2019. Malleable Games - A Literature Review on Communities of Game Modders. In The 9th International Conference on Communities \& Technologies - Transforming Communities (C\&T 2019), fune 3-7, 2019, Vienna, Austria. ACM, New York, NY, USA, 12 pages. https://doi.org/10.1145/3328320.3328393

\section{INTRODUCTION}

In addition to big data and blockchain, co-creation and participation are two buzzwords that influence how businesses operate as well as how our daily life is structured. To ensure continuing economic

Permission to make digital or hard copies of part or all of this work for personal or classroom use is granted without fee provided that copies are not made or distributed for profit or commercial advantage and that copies bear this notice and the full citation on the first page. Copyrights for third-party components of this work must be honored

For all other uses, contact the owner/author(s).

C\&T 2019, June 3-7, 2019, Vienna, Austria

(C) 2019 Copyright held by the owner/author(s)

ACM ISBN 978-1-4503-7162-9/19/06.

https://doi.org/10.1145/3328320.3328393 growth, industries rely on creative individuals that are able to come up with innovative solutions that can withstand challenges caused by increasingly dynamic social and economical structures [16, 30, 58]. This growing demand for creative individuals in the labor market raises a number of questions for society; these include the question of where to find creative individuals, how to identify those as well as how to train for creativity.

The link between creativity and play has been known for a long time now. Play as been conceptualized as "arena for the expression of creativity" $[17,65]$ and as "cradle of creativity in organizations" [42]. As such, play has been attested the ability to stimulate, facilitate and rehearse creativity. Evidence that this also applies to playing video games has recently been put forward (e.g. [10, 84]). Others have also started to make the argument that engaging in video game development projects can foster creativity [16]. We hence posit that taking a closer look at communities of people who engage both in playing video games and developing for video games in their spare time for inspiration on how to foster creativity in individuals is highly valuable.

Furthermore, playing video games is no longer an activity that only children engage in. In fact, the average age of people who play games is in the 30s. This development has further pushed the ludification of culture and given rise to the exponential growth of the economy around video games. In the last decade, the video game industry has increased from US $\$ 73.5 \mathrm{~B}$ to US $\$ 138.5 \mathrm{~B}{ }^{1}$.

Game modding has had a significant impact on the economics of game development, as can be seen with the top three games by esports gross prize pools ${ }^{2}$ are Dota 2 (US\$174M), Counter-Strike: Global Offensive (US\$69M), and League of Legends (US\$64M). A common thread across these games is that they are all commercial versions of what began as game mods, with Dota 2 and League of Legends both inspired by the Defense of the Ancients mod for the game Warcraft III by Blizzard, and Counter-Strike: Global Offensive based on Counter-Strike, a mod originally for the game Half-Life by Valve. This and the fact that game companies have begun to hire the developers of the original mods (e.g. in the case of Team Fortress Classic), illustrates the change in attitude that game companies used to display towards game modders, which went from threatening with legal lawsuits on the basis of copyright infringements to openly encouraging the creation of mods by providing the necessary tools.

Former mods ranking among the most popular and hence successful games emphasis the immense value of game modding in terms of stimulating innovative and creative ideas. Despite the

\footnotetext{
${ }^{1}$ Source: https://www.bloomberg.com/news/articles/2019-01-23/peak-video-gametop-analyst-sees-industry-slumping-in-2019

2 Source: https://www.esportsearnings.com/games
} 
millions of daily players ${ }^{3}$ and billions of yearly revenue, little is yet known about the dynamics and trends around communities of those who modify games. In order to be able to capitalize of the creative potential that these communities pose, it is essential to have a clear understanding of the hows and whys they work. We argue that approximately two decades after the release of one of the most successful game mods ${ }^{4}$, it is time to take stock on what is known about game modding communities. We hence conducted a comprehensive literature review on research related to the modification of games. In this paper, we present our findings linking them to common themes and aspects relevant to both economy and creativity. We begin by outlining our methodology for the literature search. To provide context for the following sections, we included a brief introduction of relevant terms used in the context of game modding. Subsequently, we provide an overview to findings related to meta-data of the identified literature including the research discipline the article belongs to, the type of mods and games covered. The main body of this paper presents the content-related findings of the literature review, which we structured into three sections according to the perspective the respective articles took on game modding. Before concluding the paper, we identify some potential avenues for future research.

\subsection{Methodology}

The purpose of this paper is to provide an overview of research on game modders. To accomplish this, we adopt a summarization of knowledge approach, where we provide a descriptive account of researched aspects. A secondary aim is to investigate what is known about the dynamics, interactions of and differences among game modding communities. For this purpose, we aimed for a both broad and specific review. The database of Scopus was queried as resource for our systematic literature search.

We conducted exploratory searches to ensure that used keywords would cover relevant literature. This informed our final search query:

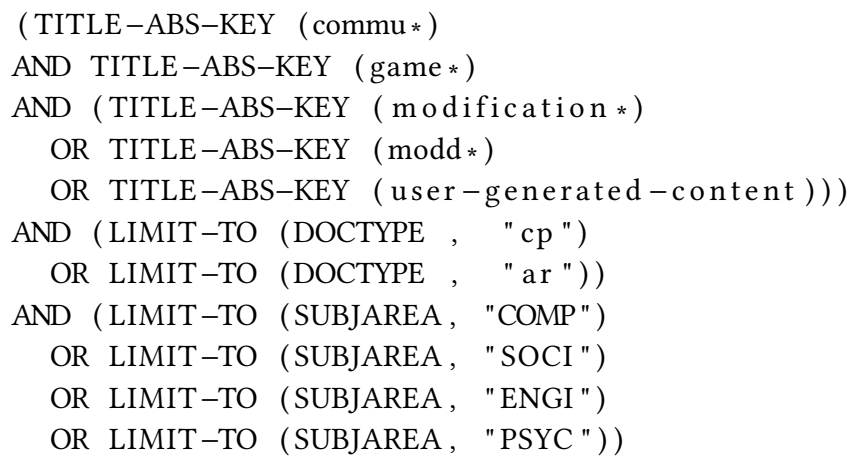

The literature search was carried out in February 2019 and resulted in the identification of 217 manuscripts. Of those 56 were deemed relevant as they touched upon aspects of game mods. The database query was complemented by checking citations and references as well as identifying related work. Forward references

\footnotetext{
${ }^{3}$ STEAM current statistics of who is playing games at the time of writing include 800.000 people playing Dota 2, and 500.000 people playing Counter-Strike: Global Offensive. Source: https://store.steampowered.com/stats/

${ }^{4}$ Counter-Strike was released in November 2000.
}

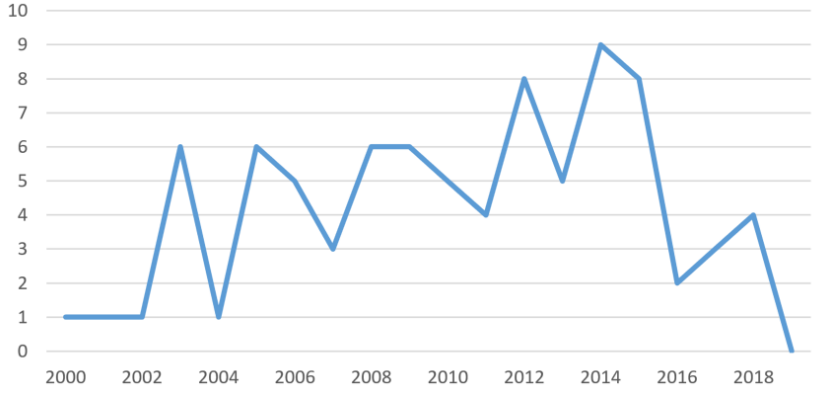

Figure 1: Graph showing the number of publications addressing topics relevant to game modding per year.

revealed 9 and backward references 1 additional publication. Using keywords and terms from the 56 relevant manuscript in other academic databases (e.g. local digital library, Google Scholar) revealed 31 additional publications.

Of those 97 manuscripts, 87 publications were of academic nature and 10 were magazines (e.g. news paper articles). The text of 3 publications was inaccessible to us and thus excluded from the fundus. 12 articles did not cover game modding despite their metadata. The analysis of our literature review is hence based on 72 academic publications.

\section{BACKGROUND}

Timeline. Researchers' interest in game modding has been going up and down in the last decade (see Fig. 1). Although we did not restrict our literature search to a specific time frame, in this section we focus only on the last decade. A definite peak of interest could be observed in the years of 2014 and 2015, but also in 2012 where eight publications related to modding were published. The reason for this peek is not entirely clear, but might be connected to the release of some rather popular game mods, one of them themed around the immensely popular book and TV-series Game of Thrones and a quite successful total conversion based on the game ArmA II named Day $Z^{5}$. In recent years, academic interest in game modding ebbed but might see new relevance under themes such as creative participatory culture (here focusing on the creativity displayed by modders) and as post-industrial economy [70, 75].

The practice of game modding seems to spark academic interest in a very broad range of research disciplines. With $64 \%$ of the identified publications being journal articles, we examined to which research discipline these could be attributed to. Our findings show that game modding is relevant to culture, game and media studies, law, customer/consumer and creativity research, arts, health and computer science. Common outlets for game modding research seem to include the journals "Games \& Culture" (9), "new media \& society" (4), "simulation \& Gaming" (3) and "Convergence" (3).

\footnotetext{
$\overline{{ }^{5} \text { Source: https://www.techspot.com/review/618-pc-game-mods-2012/commentsOffset }}$ (assessed 21.02.19)
} 
Terminology. Since this review will make reference to both a number of games and mods, it seems reasonable to include a short paragraph that explains some of the terminology used in this context. As Marone pointed out, modders make extensive use of specialist language within their communities to signal both competence as well as belonging, but also to build their identity as knowledgeable insiders [43]. Apart from insider terms, there is a plethora of types of mods that are known by specific terms. Here we provide both a brief discussion of the term "game modding" and a short (non-exhaustive) overview to provide context and later reference.

Reflecting modding as a dynamic and constantly evolving concept, there is neither an official or commonly accepted definition of modding nor an exhaustive list of all type of mods that everyone involved (i.e. gamers, modders, game industry, research) would agree on. In the course of our literature review, we came to understand that every scholar coins their own definition while occasionally borrowing from or basing it on previous ones by other scholars $[7,18]$. There does not even seem to be consensus on whether the focus of game modding is on making changes to an existing game [46, 51] or the development (i.e. addition) of new content $[8,36]$. From what we came to know about mods, we believe it is fair to say that is both [67]. To that Wallace adds that also deleting code or features from games is part of game modding [77]. What most scholars do agree on is that this modification (including development of something new) stems from users (in this context most often players) and not from professionals (at least not as part of their paid job). Others have stressed that in order to considered a mod, modifications have to be done to a commercial product $[18,36]$. At this point, we would like to point out that modifications of user-created game mods are also mods. A popular example for this is creating new maps or content for Counter-Strike, which is a mod of Valve's game Half-Life. Another addition to a potential definition of game modding, which might clarify the former distinction, is that these modifications are neither "authorised nor intended by the manufacturers" [18]. In light of a paradigmatic change in how the game industry (at least the bigger players) deal with game modding, this aspect might have lost its relevance for some types of mods (as discussed in 3.1.2). Another aspect to describing the practice of modifying games is the usage of (software development) "tools that are not part of the [original] game" [51]. This distinction excerpts content that was created with in-game tools from being considered as mods (e.g. characters created in The Sims Creator or levels designed in Lemmings). With in-game we refer to tools that are part of the main game and can be assessed from within the game without having to start another (external) program. Finally, it should be noted that the term "mods" is not exclusive to games but is also used for non-entertainment technology $[18,36,46]$. While we do not intend to introduce a new, inclusive definition for game modding, we understand mods as the product of user-made modifications, which includes the process of altering, appropriating, adding to and deleting, of video games using tools that are not part of the original game.

When it comes to mods, neither type nor complexity matters, as pointed out by Sotamaa every alteration to software no matter how small or how complex can be treated as a mod [67]. As a complete overview of all possible type of mods would go beyond the scope of this paper, we will only introduce those that are referenced in other parts of this paper. Whereas additions that are used within a game (generally know as add-ons) and modifications that affect aspects within a game (e.g. user-interface customizations) belong to one category of mods, total conversions are stand-alone games that are based on an existing game. Counter-Strike being based on Half-Life is the most popular example for this type of mod. To that end, total conversions are the most extensive type of mod as they more or less involve all types of mods [1, 67].

Apart from a few exceptions, terminology used for different types of modders is rather straight forward as the terms simply describe what kind of mod they do. For instance, mappers create maps for existing games that make use of content and mechanics of the original game, skinners create skins for new characters, objects or artefacts and level-designers design levels. Machinima is one of the less palpable terms. They also belong to another category of mods which is also referred to as fan-art. Wirman [81] categorizes fan-art as expressive form of game related fan/player productivity opposed to instrumental forms, which include walkthroughs, tutorials as well as cheat code listings. Mods as we defined them in the paragraph above are placed in the intersection of instrumental and expressive forms of user-generated content. Due to that distinction, strictly-speaking fan-art does not fall under the definition of game mods, however in light of quite a number of research related to game mods and in particular modding communities focusing on fan-art and especially machinima, we chose to still include it in this review. Machinima is an amalgamation of the words "machine" and "cinema" [25], which describes the art of using scenes and content from video games to create films. Since in order to create these films often modifications to games are necessary (explaining the link to game mods), machinima has been described as convergence of filmmaking, animation and games development [23]. One popular example of machinima that involved heavy editing and modifications is a short film based on The Sims 4 posted by a user named Rémi Marocelli is part of the series around the fictional character Bella Goth ${ }^{6}$.

Types of mods. In the course of our literature review, we were further interested in whether publications on topics related to game modding would talk about this practice more generally or focus on specific type of mods. The following list is not mutually exclusive, since some papers focused on multiple types of mods. As already indicated, a larger number of publications focused specifically on machinima [23-25, 40, 41, 57]. Others talked more general about fan-art $[15,79]$. Another type of mod that was featured quite often are levels $[9,22,43,45]$. User-interface modifications were picked as a central theme by $[62,66,74]$. Other types of mods covered by recent research relevant to game modding touched upon add-ons [62], skins [83], game rules [71] and - at the verge of the modding spectrum - tutorials [15, 35], workshops [15] and streams [31]. In fact, recently there has a great academic interest in streamers and their communities. But not being a mod, our literature search only picked up one publication (also) addressing streams.

Covered games. When wanting to explore the differences of game modding and in particular differences in game modding communities it is worth looking at the various games and genres that

\footnotetext{
6 "BELLA GOTH: THE LAST HOPE | Sims 4 Machinima" - https://www.youtube.com/ watch?v=ghCLKrNxj1g
} 
are being modded. For this section, we reviewed which games are commonly being discussed in contrast to just being mentioned in passing as examples or to illustrate a point. Within the results of our database search, most commonly discussed games are The Sims [27, 38, 64, 82] and World of Warcraft [14, 36, 62, 74]. For both games three papers each exclusively focused on modding practices related to that game. Other games that were featured in game modding research are Little Big Planet $[9,43]$, Skyrim [57, 66], Minecraft [5, 46], Super Mario [45, 48], Battlefield [14, 54], League of Legends [14], Civilization [49], Counter-Strike [83], Grand Theft Auto [38] and Dark Souls [20]. From this (non-exhaustive) list we can infer the different game genres that are being discussed in research as examples of game modding practices. First-person shooters (FPS) seem to be quite commonly featured in academic discourse as examples for game modding, so are role-playing (RPGs) and platform games. Also massive multi-player online games (MMOs) and their sub-form multi-player online battle arenas (MOBAs) are of academic interest. Conversely, we can also note which game genres have so far not received academic attention (e.g. sports, party and casual/mobile games). The vast majority of those games are designed to be played by multiple - in case of MMOs large amounts of - people at once. Seeing that is more enjoyable to play together with peers, these games already establish communities around them. Picking up on aspects that players would like to have improved or included in their favorite games as well as identifying potential mod team members is arguably easier when knowing where players of these games meet and communicate.

Methodologies. As the type of methodology applied certainly influences the validity as well as depth of insights research is able to gather, we further reviewed how the identified body of literature obtained their data regarding game modders. For some of the identified papers, it was not clear what sources they used as they nowhere specified their methodology. This might point towards the fact that game modding and mods in general are already considered common knowledge. Hence, giving a true account on what methodologies and research approaches were applied is rather difficult. With the exception of one paper [53], all papers (that specified their methodology in some way) used qualitative approaches. The majority of publications merely stated that they reviewed games, blog posts, forums or other places modders and gamers posted feedback regarding game mods. Empirical studies were also conducted to broaden our understanding of game modding, interviewing game modders was thereby the most common (9), followed by surveys $[9,26,51,74]$ and observations $[24,61,86]$. Three publications specific to have conducted ethnographic research $[3,4,86]$. Others indicated to have used a mixed-methods approach and twelve publications conducted a case study.

\section{ANALYSIS OF LITERATURE}

We categorized the manuscripts that our literature search identified into three broad categories, according to the perspective from which they address game modding (see Fig. 2). These categories are not mutually exclusive, meaning that one publication could have been addressing multiple sub-categories within the three main categories. The next section follows this structure. We begin by outlining those works that approach modding from an outside perspective

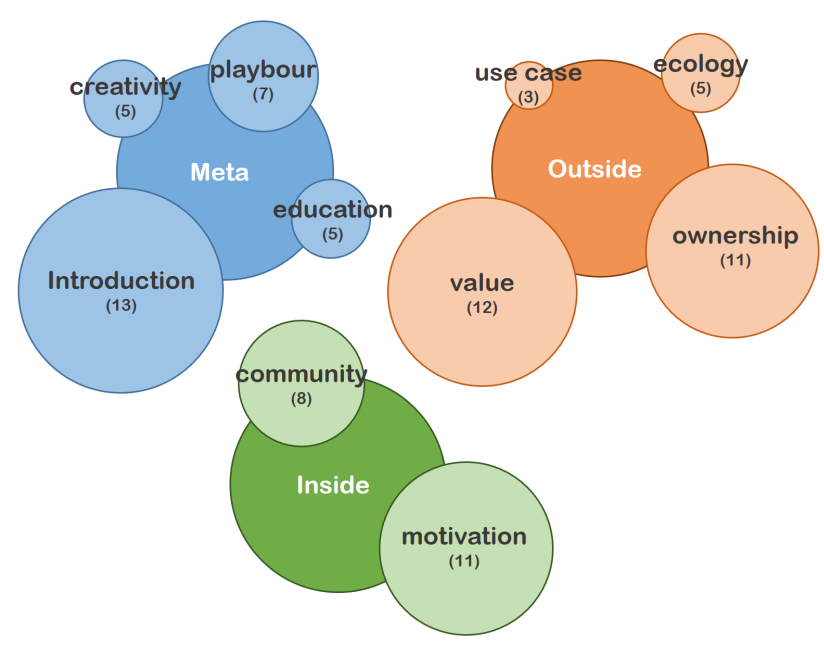

Figure 2: Overview of the literature review by categories.

describing how the practice of modifying games is perceived by entities external to game modders (e.g. game industry). We then turn towards those publications that discuss modding from a metalevel, for instance, providing overviews and case studies of game mods as well as outlining implications of modding. Finally, the inside perspective discusses aspects such as gamers' motivations to create mods and collaboration in modding communities. For each of the categories and their sub-categories we provide a brief overview of the addressed topic.

The applied categorization is not always unambiguous as some publications touch upon internal processes or outline implications for broader society and/or industry. This categorization is based on the main tenor of the individual publications.

\subsection{Outside perspective}

The largest category within the identified body of literature $\left(31^{7}\right)$ provides an outsider's perspective on game modders. Within this category, researchers mainly focused on issues regarding (legal) ownership, the value game modding can have, non-entertainment related use cases of modified games and what we termed the ecology around game mods (e.g. mapping the practice of game modding to theories and cultures).

3.1.1 Ownership debates. While modifying games certainly requires plenty of resources (e.g. time) and skills, the biggest hurdle towards creating and essentially sharing game mods has always been copyright [55]. Feeling a moral ownership over the game(s) that goes beyond legal restrictions, game modders assume a right to appropriate proprietary material [55]. Customizing and to some extent personalising games was/is important enough for most game modders to contest and even openly ignore copyright [55]. This attitude naturally led to rather emotionally charged tensions and confrontations between copyright owners and game modders, one of which resulted in a team of modders having to stop their production [55].

\footnotetext{
7 Throughout this paper numbers in parenthesis illustrate how many publications were found in a category.
} 


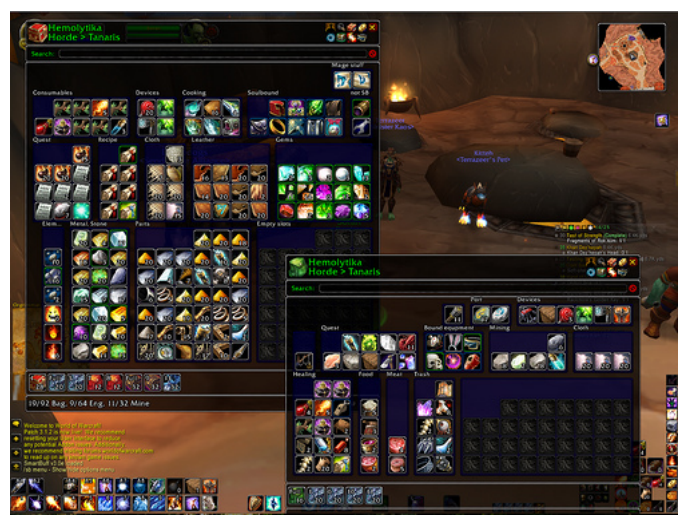

Figure 3: Example of a user-interface mod for the game World of Warcraft. The ArkInventory mod allows to sort artifacts collected during game play into self-created categories. Source https://www.flickr.com/photos/redtimmy/ 3559050961

With mods currently viewed as derivative works, modifications made without the copyright holder's consent are violations of that copyright [77]. This stance is not only contested by game modders but also individual lawyers (e.g. [1,77], who argue that mods ought to fall within the doctrine of fair use. Papers focusing on this relationship and in particular the conflict between modders and game developer companies amounts to a fair share of the body of literature on game modifications (e.g. [36, 62, 63]). It is noteworthy that even after the (admittedly slow) change of mindset regarding how to deal with game mods from being against them to openly encouraging and even facilitating them, discussions about copyright conflicts still seem to be a timely issue [32,60]. There is certainly more to be said about legal aspects that concern the modifications of proprietary games, but considering the audience of the CT conference, we will instead focus on other topics such as motivation and community.

3.1.2 Value of game mods. The above mentioned shift in game development companies' mindset regarding mods essentially entailed that the industry began to realize the value that game mods bring. Literature identified in course of this review mentions mainly three benefits of players appropriating games, namely that they (i) are good for revenue (e.g. prolong shelf-life of games; [69]), (ii) provide cheap testing grounds (e.g. exploring the acceptance of new game designs) and (iii) offer easy accessible recruiting options.

Testing new approaches through community-created mods is cheap in the sense that companies usually would have to spent huge amounts of resources on development costs (i.e. time and money). Since game modders do this work essentially for free, the industry saves these (labor) costs by having the modder community try out various designs and concepts $[29,54]$. The risk of failing then lies solely by the modders [54]. With one of their main motivation being receiving recognition for their work, it does not necessarily mean that the object of their work needs to be played a lot [51]. Getting appreciate comments that recognize the amount of work and creativity that went into the mod is sometimes enough to be worthwhile [43].
Other publications discussing the value of game mods noted that user-generated content can not only maintain interest in a game but also boost sales and hence contribute to the success of video games $[15,34]$. Anecdotely, some games players have only been waiting for its official release so they can start modding them. This new (and mostly free) source of innovation [18] can hence benefit game development both from a financial perspective - quantitatively assessed and confirmed by [53] - but also in a way of enhancing player experience [9]. Scholars have thus called for a wider and more open acceptance of players' co-creative role in game development [76].

In light of these clear benefits, mainly the larger game developing companies such as Valve and Bethesda decided to not only encourage but also enable the creation of game mods through the release of so-called software development kits (SDKs; [32]). It became apparent that the success of a game is not only to be measured by number of units sold but also by how "broadly the game acts as a medium for expression, extension, or reinforcement of a game culture" [52]. Mimicking hugely successful game concepts such as Minecraft and The Sims series that are inherently designed for active user/player participation, game companies began to release level-makers or entire game engines together with the pure game. By doing so, game companies intend to grow out of the once deprecatory attitude and adapt one that generates mutual benefit and potentially collaboration ${ }^{8}$. The provision of tools allows players to actively contribute to their own entertainment experience and give them a sense of agency and control. Yamamoto \& McArthur noted that creating the legal basis for allowing modders to sell their creations laid the foundation for constituting a new digital economy [83]. While we might not be there yet, similarities can be found in the emergence of Web2.0 and bloggers making a living out of their web presence.

3.1.3 Ecology of mods. Another body of literature attempted to understand the practice of modifying games by relating it to and mapping it in other concepts. The most common point of departure here is analyzing the relationship between modders and other stakeholders, most commonly the game industry (e.g. [7]). In 2003, Sotamaa drew connections between game modding and participatory culture, understanding the practice as a form of community-based creative design [67]. Already, scholars recognized the blurring boundaries between gamers and game designers and began to grasp the corporate culture side view on mods. In his 2010 article, Postigo sought to map modding in political economic theories and those that have understood it as participatory culture within the new media environment [56]. Banks \& Potts aimed to better understand the dualism between modding being an economic market or socio-cultural (nonmarket) force and predict which would ultimately dominate [4]. Recognizing modding as form of media commons, others reviewed whether mods are to be seen as commons or commodities [8].

\footnotetext{
${ }^{8}$ This collaboration is currently rather lopsided as the game industry receives the main share of financial outcomes (see [32]).
} 
3.1.4 Use cases. This category can be seen as somewhat an exception in the body of literature on game modding since these papers' main objective is less to understand the practice and its relationship to other cultural or economic forces, but more to illustrate application areas for game mods. Two articles, for instance, see great potential of using game and in particular their modifications for military purposes $[19,28]$. Despite the potential, Haynes also cautions for making use of it and points towards the rather realistic visualizations and the latent glorification of war [28]. A different and more pro-active approach to capitalize from game modding was proposed for the context of education. Drawing on the potential of modding to foster both learning processes and intrinsic motivation to engage with material, Dahleen et al. propose to have students modify existing serious educational games to help convey learning matter [13].

\subsection{Meta-level perspective}

Publications in this category touch upon both an outside as well as inside perspective. Their main objective is to better understand the practice of game modding by providing an overview, presenting a case study or/and studying the effects (5), impacts (5) and perceptions (7) of creating mods.

3.2.1 Introduction. This sub-category comprises identified publications that either present an overview or a case study. Speaking towards the complexity of game modding, the vast majority of overview papers focus on either a type of mod or a game genre. Focusing solely on first-person shooters, Nieborg provides an overview of current trends within the what he terms "mod culture" and based on that tries to formulate a (re-)definition of mods [47]. Small explores the connection between mods and participatory design using the example of user interface modification within and for the game Skyrim [66]. In contrast to these focused overviews, Laukkanen aims to provide a more holistic picture of game modding by outlining the case of three diverse games (Half-Life, The Sims, and Grand Theft Auto III) [38].

A distinct number of publications within this category as well as across our entire literature review focus on fan-art and in particular machinima. Apart from the popular and admittedly most successful game mod Counter-Strike as example for first-person-shooter total conversions, fan-art is most widely known also among non-gamers. This is probably due to them achieving the broadest and widest reach, seeing that they - particularly true for the case of machinima - are posted on Youtube and can be seen as form of entertainment in their own right. Whereas both Lowood and Harwood provide an overview of machinima [23], Lowood focuses on the historical development of this type of user-generated content [41]. In an attempt to compare the level of quality of professionals and amateurs, White Cheung reviewed articles and stories on fantasy sports written by game modders (fan fiction) and those by professional journalists [79]. Tapping into another category (those of effects of mods) is the case study of Owens, which points towards the potential of teaching history through and with game mods. In light of game modders valuing authenticity and attention to detail, their creations could be used to illustrate (daily) life and processes from the past as a way to facilitate education.

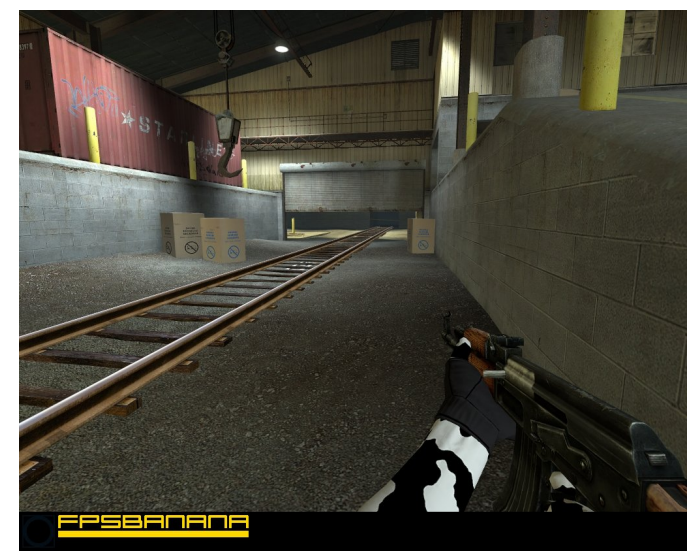

Figure 4: Example of a skin mod for the game CounterStrike created by Toxic3, who was complimented for the creative idea of re-painting the character's arms with a cow pattern. Source https://gamebanana.com/skins/19189

3.2.2 Education. Not only the products of game modding can promote education, but also the process of their creation can be seen as knowledge and skill acquisition. Although an exploratory study found only little conclusive evidence of a connection between game content creation and self-assessed IT proficiency relationships, the study authors and others still argue that engagement in game modding provides a promising approach to addressing the new digital divide [26]. In other words, they see game modding as an entry point for youth developing identities and skills as designers rather than just consumers of IT. In response to the gender distribution of gamers becoming more balanced ${ }^{9}$, Yucel et al. advocate game modding as a way to in particular attract girls to IT [85]. That this is a valid approach shows Hayes King's case study which based on interviews with female The Sims modders describes how players engage in computing practices that are foundational to information technology (IT) fluency [27]. Their findings recommend to re-orientate computer-related learning around core game modding principles, essentially linking computing to fun and by highlighting collaborative aspects frame computer-related skills and tasks such as programming as participatory design [80]. To better incorporate those principles into teaching practices, Monterrat et al. proposed a model for learning through the activity of modding [44].

3.2.3 Creativity. Previous works have identified game modding as a phenomenon that has its roots in paradigmatic changes, first and foremost Web2.0 [29, 44]. At the core of the Web2.0 ideology is the understanding that the Internet serves as platform for participation and collaboration [76]. Along with the ludification of society, fun and play further motivate what has been termed creative participatory culture where consumers actively engage in the production and recreation of content [55]. In other words, traditional consumers of information and content transform into (co-)producers of content.

The same paradigmatic change can currently be observed in the context of video games (see also 3.1.1). With games serving

\footnotetext{
9 Distribution of computer and video gamers in the United States from 2006 to 2018, by gender - https://www.statista.com/statistics/232383/ gender-split-of-us-computer-and-video-gamers/
} 
as medium of creative (self-)expression and mods slowly being recognized as significant source of innovation, game modders and their communities can be considered creative communities of practice [22]. Reflecting this, game modding has previously been defined as "creative transformation of a game's original visual content" [61] and modders representing "the archetypal creative individuals of an increasingly digital modernity" [46]. Echoing the previous argument that game modding is a form of participatory design, game mods can be seen as community-based creative design where modders are an influential source of innovation ([67]; see also 3.1.2).That ideas stemming from the player base are a force to be reckoned with is illustrated by cases such as Counter-Strike (a total conversion that became more popular than its base game Half-Life), Defense of the Ancients (DOTA; a total conversion of WarCraft 3) and Team Fortress (a total conversion of Quake).

Surely, discussing modding as creative expression warrants a discussion about what "creative" and "creativity" actually entail. The most commonly used definition of creativity describes it as the "combination of novelty and usefulness" [73] or formulated as human trait: "the capacity to create something new and original" [46]. Seeing that both "useful" and "original" are still rather subjective terms, it has been suggested to let the community or society that created the product or service in question decide on the extent of creativity involved rather than applying universal metrics [12]. This stance reflects the current debate on whether creativity can in fact be measured (e.g. with quantitative assessments) or only experienced $[11,50]$. In parallel to this discussion, scholars became to focus on how people displayed their creativity rather than evaluating outcomes of creative processes [33]. Accordingly, creative styles aim to describe how individuals facilitate creative work in their everyday lives [78]. Applied to the modding context, all forms of mods are creative products of participatory design. For some the level of creativity is more obvious than for others. For instance, from a technological and computer science point of view also what has been termed hard-core modding (e.g. ROM hacking and tinkering with physics engines) is a form of creative expression [29]. For these examples approaches have been proposed to computationally evaluate their creativity [22]. On the other end of the spectrum lie user-generated films narrating captivating stories recorded within first-person shooters or majestic castles with detailed interior design built within The Sims (cf. [64]). Recognizing the variance of skills and competence required for this creative knowledge work, scholars have even proposed to use the term phronesis ${ }^{10}$ to describe game modding [86].

3.2.4 Playbour. Along with the paradigmatic shift initiated by Web2.0 and the emergent participatory culture, more than one boundary slowly dissolves; consumers take on the role of producers [61], leisure turns into work [70] and - particularly within the game modding context - play becomes a form of content provision [25]. This section addresses the ongoing debate on whether the practice of modifying games ought to be seen (and eventually also valued) as work or leisure. As Sotamaa rightfully pointed out this discussion is neither new nor exclusive to game cultures [70].

In order to take on a holistic perspective concerning this debate, it is pertinent to not only consider the "visible", "material" labor (i.e.

\footnotetext{
${ }^{10}$ Phronesis: a type of knowledge relevant to practical action and reasoning
}

the coding, editing, designing) but also the immaterial labour in the form of creativity, communication, emotion and cooperation when talking about the "work" players put into creating game mods $[39,75]$.

The argument that all activities associated with games are play and thus non-profit-oriented [37], essentially denies modders the right to actually do try to make a living out of their hobby or at least compensate for the resources and efforts they put into their creations. Efforts that the game industry is increasingly taking advantage of $[1,64]$. It then seems even more ironic that game industries do not want their audience to associate games and thus play with "boring realities" of work contexts [70].

Some scholars are of the opinion that modding is a coercion of leisurely play into work, framing it as "playbour" [37, 70]. It seems that skeptics advancing the view that modding is a form of productive play aimed at external goals such as gaining jobs in the game industry and building a reputation for themselves, are missing the point of modding essentially being an intrinsically motivated and community oriented activity (see for a more detailed discussion on this topic). If that were to be the case, the rationale for being rather skeptical of the value of modding becomes clearer.

\subsection{Inside perspective}

Somewhat surprisingly, publications dealing with the internal dynamics and structures of game modder communities made up the smallest category (21). Two trending topics when aiming to understand the practices of game modders are their motivation (11) and community aspects (8).

3.3.1 Motivation. The undeniable benefits that mods bring for the game industry are certainly one incentive to better understand the motives behind the efforts that players engage in when creating mods. Providing for these motives by, for instance, generating affording or enabling environments (e.g. by releasing development tools) allows game developing companies to somewhat influence or steer mod development. Another rationale behind wanting to better understand modders' motivation is to better design educational tools that aim to through creating mods convey computer-relevant skills and IT proficiency more broadly.

Works investigating motives of game modders have attempted to categorize motivations. While identified motives do overlap to a great extent, the found categories do mostly not. On a very basic level, it can be said that motivations are both intrinsic and extrinsic. In the following, we attempt to provide a brief overview of the most prominent reasons for players to go through the process of producing game content themselves.

Sotamaa divides game modders into two camps; those who do it for fun/to better the game/to add content/to enjoy themselves and those who do it for personal gain/"popularity"/status [70]. While this is certainly the case, there is still dissent on which group is more prevalent. Poor found that extrinsic motives such as gaining a job in the industry are more important for less experienced compared to those who have been modding games for quite a while [51]. Again, Sotamaa's categories essentially speak to the division into intrinsically and extrinsically focused motives. If we were to take the number of facets within the two categories as any indication, intrinsic motives would certainly dominate. 


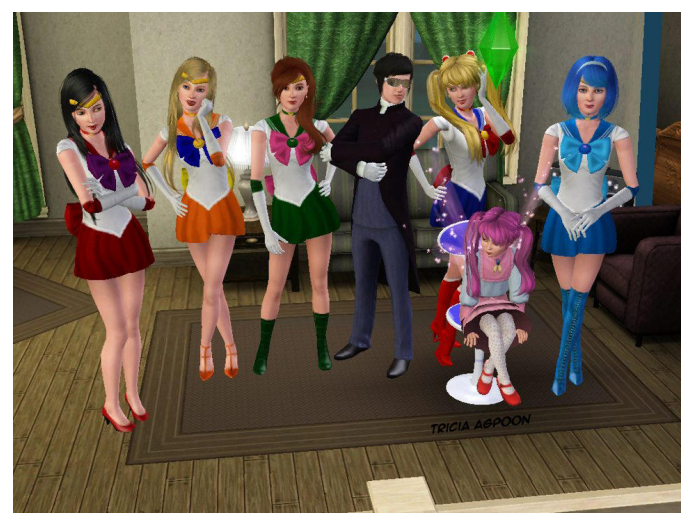

Figure 5: Example of a compilation of skin mods for The Sims 3, where interest for the game was combined with feeling nostalgic about the TV-series Sailor Moon, which the poster of this picture stated to miss watching on TV. Source https://www.deviantart.com/sugarandspice5/ art/Sailor-Moon-The-Sims-3-406760027

Intrinsic motives can be attributed to both individual and community oriented motivation. The latter comprises game modders enjoying the sense of community when sharing, collaborating, commenting on mods. (Social) interaction is immensely important for modders since it is not only a source of receiving feedback $[55,70]$ and recognition [43] for their hard work and dedication but also support [55], recommendations as well as a source of inspiration [2, 51] It is commonly agreed that modding is a highly participatory and collaborative activity [36, 44, 51, 56, 57]. Based on this strong focus on and sense of community and belonging [57], Harwood Garry saw strong parallels with tribal based communities [24]. Yet, some scholars pointed out that modders are far from being a homogeneous group and that some engage more than others while others only mod for themselves [51, 61]. In fact, it has been noted that the personal component has a stronger influence on the motivation to create mods [36, 51]. Similarly, due to some rivalries and friction, some teams have voluntarily shut themselves off from larger modding communities and, for instance, created local forums [70]. One example for a - due to various reasons - rather shut-off and isolated community of modders are those that create modifications for The Sims series (cf. [82]).

Whereas game modders used to be seen as lonely and weird "geeks", with gaming becoming ever more rooted in everyday culture and the paradigmatic shift towards "prosumerism" and participatory culture, game modders are no longer isolated but part of large communities that have an increasing influence on the game industry [55]. This empowerment of the consumer is further facilitated through wider access to technological know-how. In light of the influence these communities start to have (cf. [72]) connected with their set of norms and their specific rationale that is driven by devotion as well as the will for creative self-expression, scholars soon recognized the ecology around game modders as digital moral economy and the cultural economy of fandom [55]. This strong focus on community is not surprising, seeing that being grounded in communication play is essentially a community-reinforcing activity $[6,51]$.

Game modders' intrinsic motives can also be classified using self-determination theory (SDT; [59]), which describes three innate needs: relatedness (need to interact and be connected with others), competence (need to be effective and master problems), and autonomy (need to control one's own life). The above discussed community-focused motive certainly links to the need for relatedness. With game modding being knowledge work [75], modders are intrinsically motivated to show competence. As such they enjoy achieving (self-set) goals (e.g. completing a mod), proving (to themselves) that they can do/achieve something (e.g. changing a game mechanic; [51]). Other modders are just inherently interested in how a game "works" and the interaction of building blocks that the game is composed off [70].

The third innate need mentioned by SDT is autonomy. Connecting back to the argument that game modders perceive to have stronger connection to games than their creators (here game development companies) and claiming a moral ownership, gamers also want to exercise control over the object that they are so passionate about and hence assume a right to appropriate [55]. Having the ability and acclaimed right to change and add to games allows modders to achieve a sense of creative ownership of both the mods and the entire game [51]. Related to the motive of exercising control and creative ownership, is seeing modding as a creative outlet and the process of creating mods as artistic endeavor [54, 69].

Motives that can only partially be explained by SDT are rather specific to the game and modding context. A strong motive that also served as the theme for some publications (e.g. [2,55]) is meaningmaking [55]. As pointed out by Gee, when released games are essentially empty shells or tools that are only filled with life and meaning by play, players' experience and the emergent culture around them $[2,21]$. Meaning can come in the form of designing elements or structures that are of personal importance to the individual modder. These could be of popular (e.g. remoddeling maps according settings from Game of Thrones) or national culture (e.g. changing the theme of a game to be about Finnish wars) or - reflecting nostalgia - remind them of the past (e.g. including vehicles known from the GI Joe cartoon in Battlefield 1942) [54, 55]. Others have further noted that meaning making and hence modding is a way to make the game a bit more one's own and personal so as to better identify with it [54]. Having interests such as the ones above in common, further helps build identity and a feeling of belonging [57]. Nonetheless, Sotamaa noted that there is yet little research on the various meanings modders attach to their actions [68].

Another rather central motive for modding is the desire to improve the game and ultimately one's play experience. Modders stressed that they wanted more for the game $[8,54]$, which again illustrates their devotion to some of these game titles. This "more" could be related to better graphics, support for specific in-game features or inclusion of additional material, characters, etc. Others mentioned that for some modding is all about having fun, either as enjoyment of the products or liking to code ${ }^{11}$. Accordingly, game modding can also be perceived as just another way to relax $[51,70]$.

\footnotetext{
${ }^{11}$ We used the verb "code" here to illustrate one hobby although not every type of mod requires programming
} 


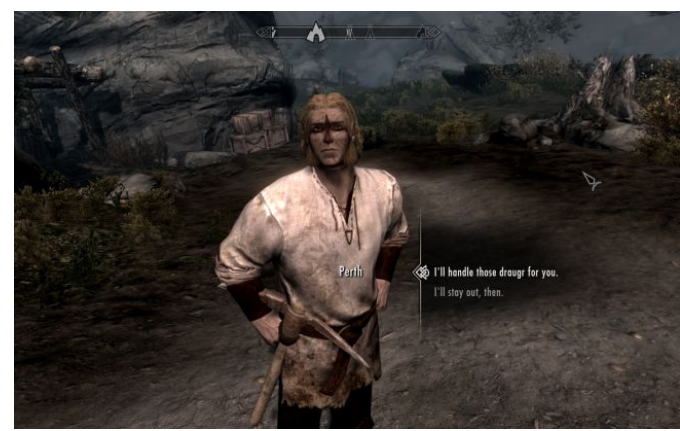

Figure 6: Mod that alters the game mechanics of Skyrim stopping auto-quests and allowing the player to customize when quests should become available, hence giving him/her more autonomy over game play. Source https://www.pcgamer. com/best-skyrim-mods/

Not to underestimate are the extrinsic motivation of game modders. One aspect that also links to intrinsic motivation but we decided to group under this category due to its relation to other extrinsic motives, is education or more broadly skill acquisition. As argued in section 3.1.2, the practice of modifying games has a strong potential of leading individuals to computer-related skills, introducing them to programming, scripting or understanding code and the internal architecture of games. In fact, modifying games requires a broad skill set that in the majority of cases modders at least to some extent taught themselves [61]. Seeing that every game and game engine is different (i.e. using different architectures and scripting languages), the process of creating a mod inherently involves learning, either in gaining more experience or mastering new skills [61]. As pointed out by Sotamaa, learning in the context of game mods is not as structured and focused as in formal education, but can better be described as "playing with the code" [70].

With the process of becoming a modders relying on self-tutoring and self-organizing, modder communities can to some extent be seen as a support network and DIY community. The exchange of information, feedback and useful tips creates a form of IT-related learning ecology [26]. At this point, it is also noteworthy that some mods such total conversions require a quite diverse set of skills ranging from programming over to visual design to editing and even choreographing videos. While mods are therefore joining forces in large teams, some modders do everything themselves. That the learning motive also has a strong intrinsic component is illustrated by some modders re-building existing content (e.g. maps) only to (a) prove that they are capable of doing so and (b) to demonstrate that things can be also be done in different ways [43, 51]. While such a re-implementation is arguably not quite novel or original, it still does exhibit creativity. This practice is yet another example supporting the need for a re-interpretation or definition of creativity in the context of game mods (cf. [43]).

Another extrinsic motive that mainly the game industry seeks to build upon is by creating game mods gain experience to acquire a job as professional game developer. Mods and mod projects they were involved would then be used as portfolio when applying for jobs. Whereas several publications mention modders' desire to get a job in the games industry as motive (e.g. $[37,54,64,70])$, the actual importance of that motive seems rather minor (cf. [51]). To that end, one has to distinguish between modders believing that based on their modding experience they would have a higher chance of getting hired and modders actually wanting to apply within the game industry. The former seems to be more rooted in modders' attitudes. Reciting a statement from one of the modders commenting on survey, some modders have a career of their own and see modding mostly as fun and relaxation [51]. Mixing modding with work for them might ruin the fun (see 3.2.4). Paradoxically, after realizing the influence game modders have on the industry and gaming culture in general [64], game developing companies increasingly view modders as potential new staff [49]. Even more paradoxical are then the efforts game industries are putting into discouraging "their audiences from associating games with boring realities as jobs and working hours" [70].

\subsection{Community}

In light of community being such a central aspect for game modders, many aspects relevant to game modding communities have already been addressed in the previous section. This section therefore summarizes themes other than motivation that were covered by publications identified by our literature review. The most common theme is hereby the relationship between modders and modding communities and what role these communities play for modders.

Given the strong sense of community among game modders and the value they attribute to both (social) interaction and collaboration [51,62], scholars have described modding communities as spaces for socializing and interaction [57]. Scholars have also described these communities as safe spaces [43] or "third places" [2, 9] where people could be more open about their interests than they would towards their offline friends [51]. Highlighting the importance of interaction and collaboration as well as creative production, Marone argues that modding communities serve as "discoursive studio" where members share and build on each others' competence to devise outcomes that will contribute to their own and other's play experience [43].

Examining machinima communities more closely, Harwood \& Garry suggest that these show many similarities with tribal based communities, but set in an online environment. One of the similarities is set also modding communities are heterogeneous networks formed by people that have a shared passion or - put more neutrally - have something in common. Further, they engage in reciprocal exchange, which could be of supportive or appreciative nature. In fact, this dialogic and democratic interaction is central to collaborative processes not only for machinima but also the development of other types of mods. Although usually not having defined hierarchies, a distinction between "leaders" and "tourists" or novices can be made within modding communities. However, game modding communities also differ from traditional tribal communities, for instance, in the sense that their members (here modders) are most often invisible as well as pseudonymous, meaning that most modders do not the true identity of their peer modders. Being aware of the exponential growth in popularity of machinima, some members of the community start to "break out" of their more or less non-profit 
but altruism oriented communities and attempt to commercialize their creations [24].

Interestingly enough, apart from two publications we found no evidence of research exploring the composition of game modding communities. While not the focus of his work, Poor mentioned the socio-demographic distribution of his survey study [51]. In contrast, Targett et al. more deliberately examined the composition of the community of those creating user-interface mods for World of Warcraft with regards to members' backgrounds, their attitudes towards mods and the community itself as well as their characteristics and motivations to create and share modifications [74]. Somewhat related to composition is Wirman's study on the role of identity in the context of game modding communities [82]. She specifically discusses the importance of gender and nationality on identity among game modding communities, pointing towards the existence of both national (local) and global game communities. She further notes that her example of The Sims might not be representative for all game modding communities as The Sims represents a special case both among gamers as well as modders.

\section{AVENUES FOR FUTURE RESEARCH}

Our literature review has not only identified content-related but also methodological opportunities for future research on game modding. Our review showed that there is still little research on the different types of modders and whether it actually makes sense to talk about "different types". To that end, there is yet little insight on whether different types of mods are exclusively being created by separate groups and types of modders or whether being a game modders more or less entails having a highly diverse range of modding-relevant skills. Similarly, some type of mods have seen less or no academic interest so far. We posit that is worthwhile to investigate whether findings related to other types of mods (and hence communities) also apply to those or whether they actually present an exception that calls for closer attention. These mods include control re-mapping, which enable players to reconfigure the default controls of a game, and modding game mechanics, such as modifying or disabling game mechanics (e.g. aiming, speed).

We further noted that while investigating communities of modders, only very few publications explored differences of game communities, but rather zoomed into specific ones and analyzed their relationship with modders. In light of game modders' proven ability to form highly productive teams that generate remarkably creative and successful outcomes which occasionally turn into products, we argue that there is value in a closer and more fine-grained analysis of how modders work both individually but especially within their communities. Such an investigation could, for instance, focus on the dynamics within modding teams and their ways and techniques of collaborating together (e.g. communication).

Regarding the methodology, there seems to be a lack of empirical research on game modding. While some aspects have been investigated using interviews and surveys, we argue that a combination of the two or more generally mixed-methods approaches are a promising way to gain a more detailed and nuanced understanding of the practice of game modding and the role communities and identities within those play. Aiming to analyze the levels and style of creativity that modders display when going about their work might warrant adopting a mixed-methods approach that includes case studies of actual mods (e.g. add-ons).

\section{CONCLUSION}

This paper presented a structured literature review of the practice of modifying video games by non-professional game developers. Focusing specifically on publications touching upon communities of game modders, might have reduced the number of identified papers and hence skewed our subset towards community-oriented topics and themes. However, seeing that we identified publications from a broad range of academic disciplines, we are confident in saying that our review provides a comprehensive overview on recent research conducted on game modding. We structured our findings based on what perspective scholars adopted when describing various aspects related to game modding. Our data shows that research has elucidated game modding from a variety of angles including an outside and inside perspective as well as addressing aspects that are on a meta-level.

As main contribution of this paper - apart from providing an extensive overview of research done in this field - we understand the identification of research gaps and hence avenues for future research. Future work from our side will entail studying more closely the role of and dynamics as well as differences between modding communities. Furthermore, we seek to gain a deeper understanding to what extent modding can be seen as a creative practice. To that end, we plan to explore creative styles and their variance, which are displayed when creating different types of mods.

\section{ACKNOWLEDGMENTS}

This project has received funding from the European Research Council (ERC) under the European Union's Horizon 2020 research and innovation programme (grant agreement No 740548).

\section{REFERENCES}

[1] 2012. Spare the Mod: In Support of Total-Conversion Modified Video Games. Harvard Law Review 125, 3 (2012), 789 - 810.

[2] Chee Siang Ang, Panayiotis Zaphiris, and Stephanie Wilson. 2010. Computer games and sociocultural play: An activity theoretical perspective. Games and Culture 5, 4 (2010), 354-380. https://doi.org/10.1177/1555412009360411

[3] John Banks and Sal Humphreys. 2008. The Labour of User Co-Creators Emergent Social Network Markets? (2008). https://doi.org/10.1177/1354856508094660

[4] John Banks and Jason Potts. 2010. Co-creating games: A co-evolutionary analysis. new media \& society 12, 2 (2010), 253-270. https://doi.org/10.1177/ 1461444809343563

[5] Beth Beggs. 2012. Minecraft, It's a Mod, Mod, Modder's World: Computer Game Modifications as Civic Discourse. Reconstruction: Studies in Contemporary Culture 12, 2 (2012).

[6] Stuart Brown and Christopher Vaughan. 2010. Play: How it Shapes the Brain, Opens the Imagination, and Invigorates the Soul. Avery, Penguin Group, New York, USA. 240 pages.

[7] Thierry Burger-Helmchen and Patrick Cohendet. 2011. User Communities and Social Software in the Video Game Industry. Long Range Planning 44, 5-6 (2011), 317-343. https://doi.org/10.1016/j.lrp.2011.09.003

[8] Sarah Coleman and Nick Dyer-Witheford. 2007. Playing on the digital commons: collectivities, capital and contestation in videogame culture. Media, culture \& society 29, 6 (2007), 934-953. https://doi.org/10.1177/0163443707081700

[9] Francesca Comunello and Simone Mulargia. 2015. User-generated video gaming: Little big planet and participatory cultures in Italy. Games and Culture 10, 1 (2015), 57-80. https://doi.org/10.1177/1555412014557028

[10] William Crombie, David C Moffat, and Olga Shabalina. 2016. Video Games can Temporarily Increase Creativity; Especially Puzzle Games. In European Conference on Games Based Learning. Academic Conferences International Limited, 152-158.

[11] Arthur J. Cropley. 2000. Defining and measuring creativity: Are creativity tests worth using? Roeper Review 23, 2 (dec 2000), 72-79. https://doi.org/10.1080/ 


\section{9}

[12] Mihaly Csikszentmihalyi. 2009. The creative person and the creative system. In Proceedings of the seventh ACM conference on Creativity and cognition. ACM, 5-6.

[13] Jacob Dahleen, Alex Hunsberger, Ryan Weber, Dennis Brylow, C.S. Shaun Longstreet, and Kendra M L Cooper. 2014. Towards a lightweight approach for modding serious educational games: Assisting novice designers. In Proceed ings of the Twentieth International Conference on Distributed Multimedia Systems. Knowledge Systems Institute Graduate School, Pittsburgh, 329-334.

[14] Michael Ebrahimi and Lei Chen. 2014. Emerging cyberworld attack vectors: Modification, customization, secretive communications, and digital forensics in PC video games. In International Conference on Computing, Networking and Communications (ICNC), 2014. IEEE Computer Society, 939-944. https://doi.org/ 10.1109/ICCNC.2014.6785463

[15] Antonia Estrella-Ramón and Fiona Ellis-Chadwick. 2017. Do different kinds of user-generated content in online brand communities really work? Online Information Review 41, 7 (2017), 954-968. https://doi.org/10.1108/OIR-08-2016-0229

[16] Carlo Fabricatore and Ximena López. 2013. Fostering Creativity Through Educational Video Game Development Projects: A Study of Contextual and Task Characteristics. Creativity Research fournal 25, 4 (2013), 418-425.

[17] Greta G Fein. 1987. Pretend play: Creativity and consciousness. In Curiosity, imagination, and play. Lawrence Erlbaum Associates, Inc, 281-304.

[18] Stephen Flowers. 2008. Harnessing the hackers: The emergence and exploitation of Outlaw Innovation. Research Policy 37, 2 (2008), 177-193.

[19] Gwenda Fong. 2006. Adapting COTS games for military experimentation. Simulation \& Gaming 37, 4 (2006), 452-465. https://doi.org/10.1177/1046878106291670

[20] Enrico Gandolfi. 2018. Enjoying death among gamers, viewers, and users: A network visualization of Dark Souls 3's trends on Twitch.tv and steam platforms. Information Visualization 17, 3 (2018), 218-238. https://doi.org/10.1177/ 1473871617717075

[21] James Paul Gee. 1999. An introduction to discourse analysis: Theory and method New York.

[22] K Grace and M L Maher. 2014. Towards computational co-creation in modeling communities. In Experimental Artificial Intelligence in Games: Papers from the AIIDE Workshop, Vol. WS-14-16. AI Access Foundation, 15-20

[23] Tracy Harwood. 2011. Towards a manifesto for machinima. Fournal of Visual Culture 10, 1 (2011), 6-12. https://doi.org/10.1177/1470412910391547

[24] Tracy Harwood and Tony Garry. 2009. Infiltrating an e-Tribe: Marketing within the Machinima [Computerised Games] Community. fournal of Customer Behaviour 8, 1 (2009), $67-83$.

[25] Tracy Harwood, Tony Garry, and Mike Uwins. 2015. Machinima: Extending brand reach. Fournal of Consumer Behaviour 14, 6 (2015), 378-388. https://doi. org/10.1002/cb.1552

[26] Elisabeth Hayes. 2008. Game content creation and IT proficiency: An exploratory study. Computers \& Education 51, 1 (2008), 97-108. https://doi.org/10.1016/j. compedu.2007.04.002

[27] Elisabeth R Hayes and Elizabeth M King. 2009. Not just a dollhouse: What The Sims2 can teach us about women's IT learning. On the Horizon 17, 1 (2009), 60-69. https://doi.org/10.1108/10748120910936153

[28] Cynthia Haynes. 2006. Armageddon Army Playing God, God Mode Mods, and the Rhetorical Task of Ludology. Games and Culture 1, 1 (2006), 89-96. https: //doi.org/10.1177/1555412005281909

[29] Renyi Hong. 2013. Game Modding, Prosumerism and Neoliberal Labor Practices. International fournal of Communication 7, 19 (2013), 984-1002.

[30] Scott G Isaksen, Kenneth J Lauer, Goran Ekvall, Alexander Britz, S G Isaksen, K J Lauer, G Ekvall, A Britz, Fainstitute Stockholm, and Sweden Alexander Britz. 2001. Perceptions of the Best and Worst Climates for Creativity: Preliminary Validation Evidence for the Situational Outlook Questionnaire. Creativity Research fournal 13, 2 (2001), 1040-419. https://doi.org/10.1207/

[31] Adele LU Jia, Dick H J Epema, Alexandru Iosup, D H J Epema, and A Iosup. 2016 When Game Becomes Life: The Creators and Spectators of Online Game Replays and Live Streaming. ACM Transactions on Multimedia Computing, Communications, and Applications 12, 4 (2016), 1-24. https://doi.org/10.1145/2957750

[32] Daniel James Joseph. 2018. The Discourse of Digital Dispossession: Paid Modifications and Community Crisis on Steam. Games and Culture 13, 7 (2018), 690-707. https://doi.org/10.1177/1555412018756488

[33] Chad J Keller, Lea A Lavish, and Chris Brown. 2007. Creative styles and gender roles in undergraduates students. Creativity Research fournal 19, 2-3 (2007) 273-280.

[34] Stefan Koch and Michael Bierbamer. 2016. Opening your product: impact of user innovations and their distribution platform on video game success. Electronic Markets 26, 4 (nov 2016), 357-368. https://doi.org/10.1007/s12525-016-0230-5

[35] Johannes Konert, Nico Gerwien, Stefan Göbel, and Ralf Steinmetz. [n. d.]. Academic Bookshop, 319-328.

[36] Y.M. M Kow and B. Nardi. 2010. Who owns the mods? First Monday 15, 5 (2010) https://doi.org/10.5210/fm.v15i5.2971

[37] Julian Kücklich. 2005. Precarious Playbour: Modders and the Digital Games Industry. fibreculture 5, 1 (2005)
[38] Tero Laukkanen. 2005. Modding scenes Introduction to user-created content in computer gaming. Technical Report. University of Tampere Hypermedia Laboratory, Tampere. 152 pages. http://tampub.uta.fi/tup/951-44-6448-6.pdfhttp: //www.uta.fi/hyper/

[39] Maurizio Lazzarato. 1996. Immaterial labor. Radical thought in Italy: A potential politics 1996 (1996), 133-47.

[40] Henry Lowood. 2005. Real-time performance: Machinima and game studies. The International Digital Media \& Arts Association fournal 2, 1 (2005), 10-17.

[41] Henry Lowood. 2006. High-performance play: The making of machinima. fournal of Media Practice 7, 1 (2006), 25-42. https://doi.org/10.1386/jmpr.7.1.25/1

[42] Charalampos Mainemelis and Sarah Ronson. 2006. Ideas are born in fields of play: Towards a theory of play and creativity in organizational settings. Research in Organizational Behavior 27 (2006), 81-131.

[43] Vittorio Marone. 2015. From discussion forum to discursive studio: Learning and creativity in design-oriented affinity spaces. Games and Culture 10, 1 (2015), 81-105. https://doi.org/10.1177/1555412014557328

[44] Baptiste Monterrat, Elise Lavoué, Sébastien George, and Élise Lavoué. 2012. Learning Game 2.0: Support for Game Modding as a Learning Activity. In Priceedings of 6th Conference on Games Based Learning. Cork, Ireland, 340-347. https://hal.archives-ouvertes.fr/hal-00738749

[45] James Newman. 2018. Kaizo Mario Maker: ROM hacking, abusive game design and Nintendo's Super Mario Maker. Convergence: The International fournal into New Media Technologies 24, 4 (2018), 339-356. https://doi.org/10.1177/ 1354856516677540

[46] Josef Nguyen. 2016. Minecraft and the Building Blocks of Creative Individuality. Configurations 24, 4 (2016), 471-500. https://doi.org/10.1353/con.2016.0030

[47] David B Nieborg. [n. d.]. Am I Mod or Not?-An analysis of First Person Shooter modification culture. In Creative Gamers Seminar - Exploring Culture in Gaming. Tampere.

[48] Casey O'donnell. 2013. Wither Mario Factory?: The role of tools in constructing (co)creative possibilities on video game consoles. Games and Culture 8, 3 (2013), 161-180. https://doi.org/10.1177/1555412013493132

[49] Trevor Owens. 2011. Modding the history of science: Values at play in modder discussions of sid meier's civilization. Simulation and Gaming 42, 4 (2011), 481495. https://doi.org/10.1177/1046878110366277

[50] Davide Piffer. 2012. Can creativity be measured? An attempt to clarify the notion of creativity and general directions for future research. Thinking Skills and Creativity 7, 3 (2012), 258-264.

[51] Nathaniel Poor. 2014. Computer game modders' motivations and sense of community: A mixed-methods approach. new media \& society 16, 8 (2014), 1249-1267. https://doi.org/10.1177/1461444813504266

[52] Cindy Poremba. 2003. Remaking Each Other's Dreams: Player Authors in Digital Games. In Proceedings of New Forms Festival Ât03. Canada.

[53] Leo Lev Poretski and Ofer Arazy. 2017. Placing value on community co-creations: A study of a video game 'modding' community. In Proceedings of the 2017 ACM Conference on Computer Supported Cooperative Work and Social Computing CSCW'17. Association for Computing Machinery, New York, New York, USA, 480-490. https://doi.org/10.1145/2998181.2998301

[54] Hector Postigo. 2007. Of Mods and Modders: Chasing Down the Value of FanBased Digital Game Modifications. Games and Culture 2, 4 (2007), 300-313. https://doi.org/10.1177/1555412007307955

[55] Hector Postigo. 2008. Video Game Appropriation through Modifications Attitudes Concerning Intellectual Property among Modders and Fans. Convergence 14, (2008), 59-74. https://doi.org/10.1177/1354856507084419

[56] Hector Postigo. 2010. Modding to the big leagues: Exploring the space between modders and the game industry. First Monday 15, 5 (2010).

[57] Héctor Puente and Susana Tosca. 2013. The social dimension of collective storytelling in Skyrim. In Proceedings of DiGRA 2013: DeFragging Game Studies. Digital Games Research Association (DiGRA).

[58] Mark A. Runco. 2004. Everyone has creative potential. In Creativity: From potential to realization, R. J. Sternberg, E. L. Grigorenko, and J. L. Singer (Eds.). American Psychological Association, Washington, 21-30. https://doi.org/10. 1037/10692-002

[59] Richard M Ryan and Edward L Deci. 2000. Self-Determination Theory and the Facilitation of Intrinsic Motivation, Social Development, and Well-Being. The American psychologist 55, 1 (2000), 68-78.

[60] Katharine Sarikakis, Claudia Krug, and Joan Ramon J.R. R Rodriguez-Amat. 2017. Defining authorship in user-generated content: Copyright struggles in The Game of Thrones. New Media and Society 19, 4 (2017), 542-559. https://doi.org/10. $1177 / 1461444815612446$

[61] Walt Scacchi. 2010. Computer game mods, modders, modding, and the mod scene. First Monday 15, 5 (2010).

[62] Lee Sherlock. 2009. Genre, activity, and collaborative work and play in world of warcraft: Places and problems of open systems in online gaming. Fournal of Business and Technical Communication 23, 3 (2009), 263-293. https://doi.org/10. $1177 / 1050651909333150$ 
[63] Benjamin J. Siders and Kirk A. Damman. 2015. Emerging Challenges in Tabletop Gaming: Player Modifications, Third-Party Parts, and Disruptive Technology. Vol. 7. 3605-3610 pages. https://doi.org/10.1073/pnas.0637711100

[64] Tanja Sihvonen. 2001. Players unleashed! : modding The Sims and the culture of gaming. Amsterdam University Press. 221 pages. https://www-jstor-org.ez. statsbiblioteket.dk:12048/stable/j.ctt46mt37

[65] Dorothy G Singer and Jerome L Singer. 1990. The house of make-believe: Children's play and the developing imagination. Harvard University Press.

[66] Rebekah Small. 2018. Mods and convergence culture: Connecting character creation, user interface, and participatory design. In Proceedings of SIGDOC 2018 - 36th ACM International Conference on the Design of Communication. https: //doi.org/10.1145/3233756.3233943

[67] Olli Sotamaa. 2003. Computer Game Modding, Intermediality and Participatory Culture. new media (2003), 1-26. https://people.uta.fi/\{ $\}$ olli.sotamaa/documents/ sotamaa\{_\}participatory\{_\}culture.pdf

[68] Olli Sotamaa. 2004. Playing It My Way? Mapping The Modder Agency. In Internet Research Conference 5.0. Sussex, England.

[69] Olli Sotamaa. 2005. "Have Fun Working with Our Product!": Critical Perspectives On Computer Game Mod Competitions. In Proceedings of DiGRA 2005 Conference. $1-10$.

[70] Olli Sotamaa. 2007. On modder labour, commodification of play, and mod competitions. First Monday 12, 9 (2007).

[71] Olli Sotamaa and Jaakko Stenros. 2019. Through a Shot Glass, Darkly: The Study of Games in the Light of Drinking Games. Games and Culture 14, 1 (2019), 87-103. https://doi.org/10.1177/1555412016679772

[72] Kurt D Squire and Levi Giovanetto. 2008. The higher education of gaming. E-Learning and Digital Media 5, 1 (2008), 2-28.

[73] Morris I. Stein. 1953. Creativity and Culture. The fournal of Psychology 36, 2 (1953), 311-322. https://doi.org/10.1080/00223980.1953.9712897

[74] S Targett, V Verlysdonk, H J Hamilton, and D Hepting. 2012. A study of user interface modifications in world of warcraft. Game Studies 12, 2 (2012).

[75] Tiziana Terranova. 2000. Free labor: Producing culture for the digital economy. Social Text 18, 2 (2000), 33-58.

[76] Daniel Volk. 2008. Co-Creative Game Development in a Participatory Metaverse In Proceedings of the Tenth Anniversary Conference on Participatory Design 2008. ACM, Bloomington, Indiana, 262-265.

[77] Ryan Wallace. 2014. Modding: Amateur Authorship and How the Video Game Industry is Actually Getting It Right. Brigham Young University Law Review 1 (2014), 219-255.

[78] Ming-Zhong Wang, Wu Chen, Chen Zhang, and Xue-Li Deng. 2017. Personality types and scholarly creativity in undergraduate students: The mediating roles of creative styles. Personality and Individual Differences 105 (2017), 170-174 https://doi.org/10.1016/j.paid.2016.09.050

[79] R. White and M. Cheung. 2015. Communication of fantasy sports: A comparative study of user-generated content by professional and amateur writers. IEEE Transactions on Professional Communication 58, 2 (2015), 192-207. https://doi. org/10.1109/TPC.2015.2430051

[80] Niall Winters and Yishay Mor. 2008. IDR: A participatory methodology for interdisciplinary design in technology enhanced learning. Computers \& Education 50, 2 (2008), 579-600.

[81] Hanna Wirman. 2009. On productivity and game fandom. Transformative Works \& Cultures 3 (2009).

[82] Hanna Wirman. 2014. Gender and Identity in Game-Modifying Communities. Simulation \& Gaming 45, 1 (2014), 70-92. https://doi.org/10.1177/ 1046878113519572

[83] Kei'Ichiro Yamamoto and Victoria McArthur. 2015. Digital economies and trading in counter strike global offensive: How virtual items are valued to real world currencies in an online barter-free market hybrid economy. In Games Entertainment Media Conference (GEM). IEEE, 1-6. https://doi.org/10.1109/GEM.2015.7377220

[84] Chloe Shu-Hua Yeh. 2015. Exploring the effects of videogame play on creativity performance and emotional responses. Computers in Human Behavior 53 (2015), 396-407. https://doi.org/10.1016/J.CHB.2015.07.024

[85] Ibrahim Yucel, Joseph Zupko, and Magy Seif El-Nasr. 2006. IT education, girls and game modding. Interactive Technology and Smart Education 3, 2 (2006), 143-156. https://doi.org/10.1108/17415650680000059

[86] Peter Zackariasson, Alexander Styhre, and Timothy L. Wilson. 2006. Phronesis and Creativity: Knowledge Work in Video Game Development. Creativity and Innovation Management 15, 4 (sep 2006), 419-429. https://doi.org/10.1111/j. 1467-8691.2006.00400.x 